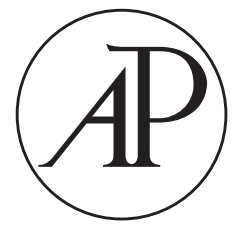

Available online at www.sciencedirect.com

science@direct.

Mechanical Systems

and

Mechanical Systems and Signal Processing 18 (2004) 59-78

\title{
An improved updating parameter selection method and finite element model update using multiobjective optimisation technique
}

\author{
Gyeong-Ho Kim, Youn-Sik Park* \\ Department of Mechanical Engineering, Center for Noise and Vibration Control, Korea Advanced Institute of Science and \\ Technology, Yuseong-Gu, Daejeon 305-701, South Korea
}

Received 25 January 2002; received in revised form 28 October 2002; accepted 11 March 2003

\begin{abstract}
Finite element model updating is a procedure to minimise the differences between analytical and experimental results and is usually posed as an optimisation problem. In model updating process, one requires not only satisfactory correlations between analytical and experimental results, but also maintaining physical significance of updated parameters. For this purpose, setting up of an objective function and selecting updating parameters are crucial steps in model updating. These require considerable physical insight and usually trial-and-error approaches are common to use. In conventional model updating procedures, an objective function is set as the weighted sum of the differences between analytical and experimental results. But the selection of the weighting factors is not clear since the relative importance among them is not obvious but specific for each problem. In this work, multiobjective optimisation technique is introduced to extremise several objective terms simultaneously. Also the success of finite element model updating depends heavily on the selection of updating parameters. In order to avoid an illconditioned numerical problem, the number of updating parameters should be kept as small as possible. Such parameters should be selected with the aim of correcting modelling errors and modal properties of interest should be sensitive to them. When the selected parameters are inadequate, then the updated model becomes unsatisfactory or unrealistic. An improved method to guide the parameter selection is suggested. (C) 2003 Elsevier Ltd. All rights reserved.
\end{abstract}

\section{Introduction}

Finite element (FE) model is very useful for design, development, and application phase of mechanical structures in target. However, results obtained from FE model often differ with test

\footnotetext{
*Corresponding author. Tel.: + 82-42-869-3020; fax: + 82-42-869-8220.

E-mail address: yspark@sorak.kaist.ac.kr (Y.-S. Park).
} 
results. There have been many attempts to correct FE model. FE model updating aims at the correction of an initial FE model that predicts accurate and reliable dynamic behaviour of a mechanical structure. The model updating problem has been investigated thoroughly and many techniques have been proposed [1,2]. However, it still remains a difficult problem [3,4]. That is because model updating is an inverse process and often contains highly non-linear characteristics. The updating methods may be divided into two groups: whether they adjust the mass and stiffness matrices directly (direct methods) or make parametric changes to the model (parametric or iterative methods). It has been shown that direct methods are not appropriate to model updating since updated results are seldom physically meaningful although these methods are capable of replicating experimental natural frequencies and mode shapes. The updating technique considered in this paper is an iterative method. One common approach of iterative methods is to consider an objective function that quantifies the differences between analytical and experimental results [5]. It is common to adjust the selected parameters to minimise the objective function, then it becomes a typical optimisation problem.

In model updating process, one requires not only satisfactory correlations between analytical and experimental results but also maintaining physical significance of updated parameters. Thus, setting up of an objective function and selecting updating parameters are crucial steps in model updating. They require deep physical insight and trial-and-error approaches are common. Fig. 1 explains their importance.

- Space $S_{1}$ contains all the possible FE models of a structure.

- Space $S_{2}$ contains all the FE models that correlate well with experimental results. One of these models, $F E_{\text {opt }}$ gives the best possible description of dynamic behaviour of the structure.

- Space $S_{3}$ is a set of models that can be derived from the initial FE model, $F E_{\text {init, }}$ by varying the selected updating parameters. Both the initial FE model, $F E_{\text {init }}$, and the updated model, $F E_{u}$, are the members of $S_{3}$.

The dimension of $S_{3}$ is determined from the initial model, which is deeply related with the selection of updating parameters. A bad selection of updating parameters will result if $S_{3}$ does not have a common space with $S_{2}$ (Fig. 1(a)). As a consequence, the updated model having good correlation with experimental results cannot be obtained even if an objective function is properly set up. Conversely, a very good selection of updating parameters will give $F E_{\text {opt }}$ within the common space of $S_{2}$ and $S_{3}$ (Fig. 1(b)). Then, whether $F E_{u}$ will converge to $F E_{\text {opt }}$ depends mainly
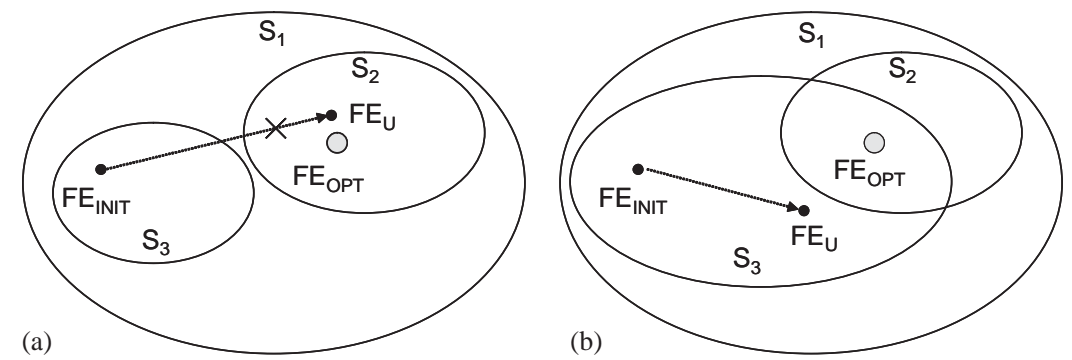

Fig. 1. Schematics of two difficulties in FE model updating: (a) poor selection of updating parameters; (b) poor set-up of an objective function. 
on used optimisation procedures. But, a poor set-up of an objective function will not guarantee $F E_{u}$ moves to $F E_{\text {opt }}$.

Since model updating is an inverse process, one can hardly classify the causes of poor updated results. These may come from a poor selection of updating parameters or an inappropriate objective function or both. Thus, when updated results are not satisfactory, the model updating optimisation process should be solved repeatedly with a modified objective function and with a different set of updating parameters until appropriate results are derived. Unfortunately, there are many alternatives for a complicated FE model. So model updating process often ends with unsatisfactory results.

In this paper, two systematic approaches are suggested to relieve the difficulties in model updating. The first part of this paper describes multiobjective optimisation technique; some important concepts related to multiobjective optimisation, the benefits of multiobjective optimisation in comparison with single-objective optimisation in model updating. The second part addresses the problem of updating parameter selection. Its importance is demonstrated through case studies and a procedure for selecting a proper updating parameter set is presented. The two approaches are seamlessly incorporated into a model updating procedure and the procedure is tested for FE model updating of a real complex structure.

\section{Multiobjective optimisation}

In conventional model updating techniques, an objective function to be minimised is usually set as a penalty function involving the weighted sum of the differences between analytical and experimental results such as natural frequencies, mode shapes, or FRF data. Although the ability to weight the different data seems versatile, the selection of the weighting factors is very difficult since the relative importance among the data is not obvious but specific for each problem. For example, the fundamental natural frequency difference may be overweighted in the objective function such that the other updated differences such as the 2nd natural frequency, mode shapes do not fit satisfactorily or vice versa. It usually takes a very long time to finally obtain satisfactory weights. The second important disadvantage of the weighting method is "duality gap" for nonconvex problems. So, an analyst might lose the opportunity to find more acceptable solutions. In this paper, the multiobjective optimisation technique is introduced to overcome the difficulties.

\subsection{Basic concepts}

Single-objective optimisation algorithms are designed to minimise (or maximise) a single criterion and work well in many real problems. But frequently, there are cases when several criteria should be considered simultaneously and it is not proper to combine these into a single term. When this is the case, the problem is said to be a multiobjective or multicriteria optimisation problem. In single-criterion optimisation, the notion of optimality scarcely needs any explanation. The best (highest or lowest) value of an objective function will be the target. In multiobjective optimisation, the notion of optimality is not at all obvious. This can be explained by the concept of Pareto optimality [6]. 
Suppose minimising both $F_{1}$ and $F_{2}$. For example, these can be the differences between analytical and experimental natural frequencies. Suppose further that we have five possible $F_{1}$ and $F_{2}$ values:

$$
\boldsymbol{A}=(2,10), \quad \boldsymbol{B}=(4,6), \quad \boldsymbol{C}=(8,4), \quad \boldsymbol{D}=(9,5), \quad \boldsymbol{E}=(7,8) .
$$

These data are plotted in Fig. 2. Among the five points, $\boldsymbol{A}, \boldsymbol{B}$, and $\boldsymbol{C}$ seem good choices even though none of the three points is the best along both dimensions. There are trade-offs from one of these three to another; there is some gain along one dimension with some loss along the other. In optimisation terminology these three points are non-dominated because there are no points better than these in every criterion. On the other hand $\boldsymbol{D}$ and $\boldsymbol{E}$ seem poor choices. This is so because both are dominated by another point. $\boldsymbol{E}(7,8)$ is dominated by $\boldsymbol{B}(4,6)$, because $4<7$ and $6<8$. And $\boldsymbol{D}(9,5)$ is dominated by $\boldsymbol{C}(8,4)$, because $8<9$ and $5<9$. Thus, in this problem instead of obtaining a single answer, the Pareto-optimal set, which is a set of answers that are not dominated by any others, is obtained.

In general, a multiobjective optimisation problem is to determine a vector of design variables within a feasible region to optimise a vector of objective functions that are usually in conflict with each other. The problem formulation in a standard form is as follows:

$$
\begin{array}{ll}
\text { minimise } & \boldsymbol{F}(\boldsymbol{x})=\left\{F_{1}(\boldsymbol{x}), F_{2}(\boldsymbol{x}), \ldots, F_{n}(\boldsymbol{x})\right\} \\
\text { subject to } & \mathbf{g}(\mathbf{x}) \leqslant 0 \\
& \mathbf{x}_{\min } \leqslant \mathbf{x} \leqslant \mathbf{x}_{\max },
\end{array}
$$

where $\boldsymbol{x}$ is a vector of design variables, $F_{i}(\boldsymbol{x})$ is the $i$ th objective function, and $\boldsymbol{g}(\boldsymbol{x})$ is a constraint vector.

The solution for a multiobjective optimisation problem is always situated on its Pareto-optimal set. A feasible vector $\boldsymbol{x}^{\star}$ is a Pareto optimum (or non-dominated) for Eq. (2) if and only if there exist no feasible vector $\boldsymbol{x}$ such that for all $i \in\{1,2, \ldots, n\}$

$$
F_{i}(\boldsymbol{x}) \leqslant F_{i}\left(\boldsymbol{x}^{\star}\right),
$$

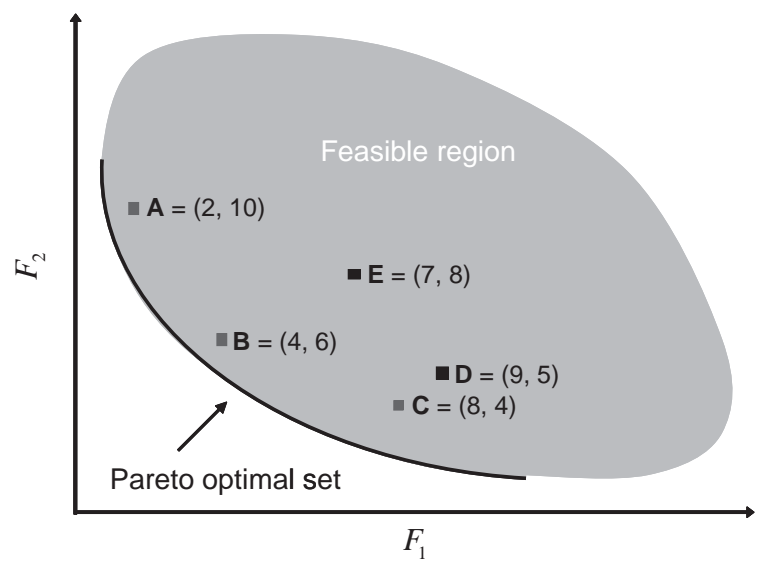

Fig. 2. Illustration of multiobjective optimisation, Pareto-optimal solutions. 
and for at least one $i \in\{1,2, \ldots, n\}$,

$$
F_{i}(\boldsymbol{x})<F_{i}\left(\boldsymbol{x}^{\star}\right) \text {. }
$$

A multiobjective optimisation gives a set of non-dominated solutions, i.e. solutions for which no objective can be improved without worsening at least one other criterion. Fig. 2 shows a minimisation problem with two objective functions where the solid line indicates the Paretooptimal set in the objective space. A typical characteristic of a multiobjective optimisation problem is the absence of a unique point that would optimise all criteria simultaneously. Any point in the Pareto-optimal set can become an optimum solution. One can make his choice on the basis of trade-offs among design objectives.

\subsection{Pareto genetic algorithm for multiobjective optimisation}

Consider the following unconstrained optimisation problem to minimise a single-objective function:

$$
\begin{array}{ll}
\text { minimise } & F(\boldsymbol{x}) \\
\text { subject to } & \mathbf{x}_{\min } \leqslant \mathbf{x} \leqslant \mathbf{x}_{\max } .
\end{array}
$$

Genetic algorithms (GAs) for single-objective optimisation use random processes to produce an initial finite population within the specified design variable space. For each member of the initial population, the objective function is calculated and then a fitness value is assigned according to the calculated value. For a minimisation problem like Eq. (5), the highest fitness value is assigned to the member that minimises the objective function. And then, three genetic operators (reproduction, crossover, and mutation) are applied to the initial population to generate successive populations that improve their fitness values over generations.

GAs search using a population of points, rather than using a point-to-point search. This search process meets the requirement of seeking Pareto-optimal points. Many multiobjective GAs have been developed to solve a general multiobjective optimisation problem like Eq. (2). The developed multiobjective GAs are essentially simple extensions of GAs treating a multiobjective function. Among them, the Pareto $G A$ developed by Cheng and Li [7] is used in this model updating recognising its effectiveness in generating Pareto-optimal points. In the Pareto GA, a penalty function technique is adopted to transform a constrained optimisation problem to an unconstrained one. As noted, in a GA for single-objective optimisation, the fitness value of a member is directly related to its objective function value. In the Pareto GA dealing with a vector function space, the fitness value of a member depends on its rank and the rank of a member depends on its non-dominated nature. Ranking a population is a continuous labelling process. At each generation, non-dominated members are identified and assigned rank 1. From the remaining population, non-dominated points are identified and assigned rank 2 . This process continues until the entire population is ranked. Members of lower rank have higher fitness value. Fig. 3 illustrates ranking for a two-objective minimisation problem. After a fitness value is assigned for each member, the three genetic operators are applied to the current population to generate a new population. Thus, the Pareto GA is essentially the same as a GA except for the fitness assignment process. In the Pareto GA, a new operator called Pareto-set filter is introduced. At each generation, the members designated rank 1 are put into the filter. When new members are added 


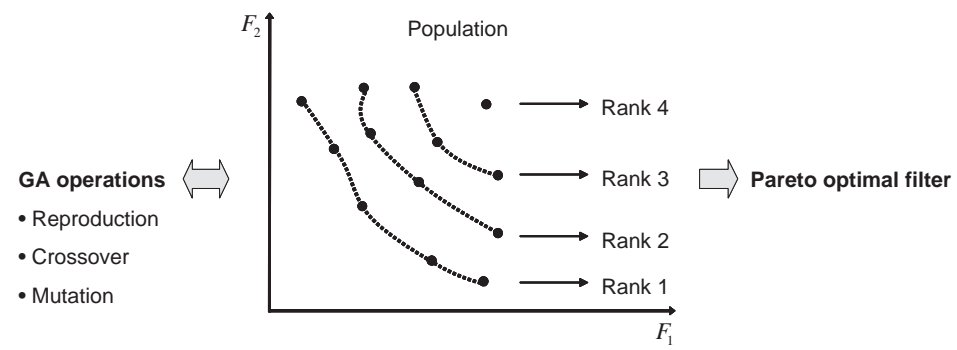

Fig. 3. Schematic of the Pareto GA for a two-objective minimisation problem (ranking, Pareto-optimal filter).

to the Pareto-set filter, all the members in the filter are subjected to non-dominated checks (filtering process) and dominated members are discharged. Thus, only non-dominated solutions are maintained in the filter. As evolution continues, the members in the Pareto-set filter go to its Pareto-optimal set zone. Fig. 3 schematically illustrates the Pareto GA for a two-objective minimisation problem.

\section{Updating parameter selection}

Since a realistic FE model has many FEs, it is impractical to associate an updating parameter with each FE. This is because the updated parameter values of neighbouring elements can be oscillatory, which are physically meaningless. And in order to avoid an ill-conditioned numerical problem, the number of updating parameters should be kept as small as possible. A popular alternative approach is to assign an updating parameter to a group of FEs, a substructure. Depending on the combinations of individual FEs, there can appear numerous substructures or updating parameters. It is a crucial step in model updating to select effective updating parameters from many combinations $[8,9]$. Parameters should be selected with the aim of correcting modelling errors, and the selected parameters should be sensitive to them. In this section, the importance of updating parameters is illustrated through simulated case studies and a procedure for selecting appropriate parameters is suggested.

\subsection{Case study}

A simple plate with a crack is provided to demonstrate the effects of updating parameter selections on updated results (Fig. 4(a)). To simulate experimental data, a fine FE model, having 466 shell elements with 3126 degrees of freedom (d.o.f.'s), is constructed (Fig. 4(b)). It is assumed that out-of-plane (z-direction) vibrations are measured at 36 points. The experimental mode shapes are plotted in Fig. 5. Fig. 6 shows an initial FE model having 114 shell elements with 840 d.o.f.'s. The corresponding mode shapes are plotted in Fig. 7. Due to the crack of the test plate, the modal properties from the initial FE model show deviations from those of the test model as summarised in Table 1 . The 2 nd and 3 rd mode pairs are poorly correlated and the initial model needs to be updated for better correlation. 
(a)

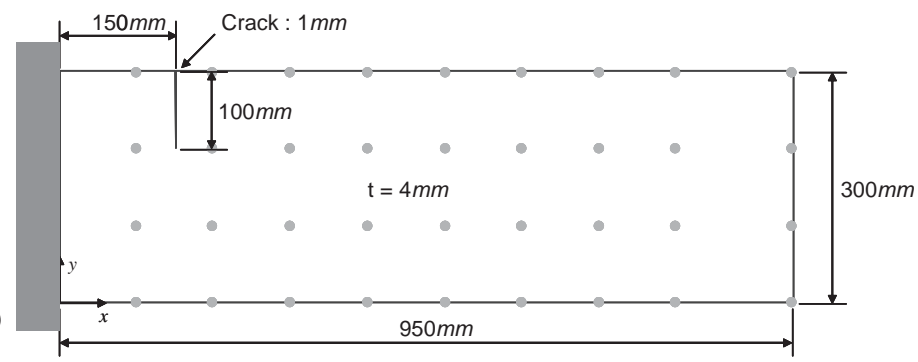

(b)

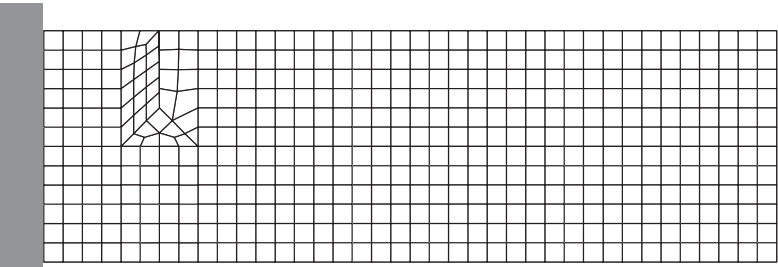

Fig. 4. Test plate with a crack: (a) geometric dimensions and vibration measurement points; (b) fine FE model (466 elements, 3126 d.o.f.'s).

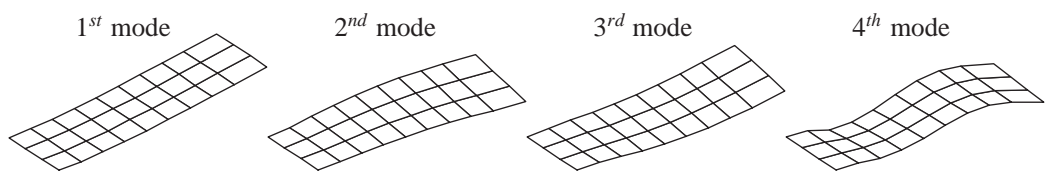

Fig. 5. Experimental mode shapes of test plate.

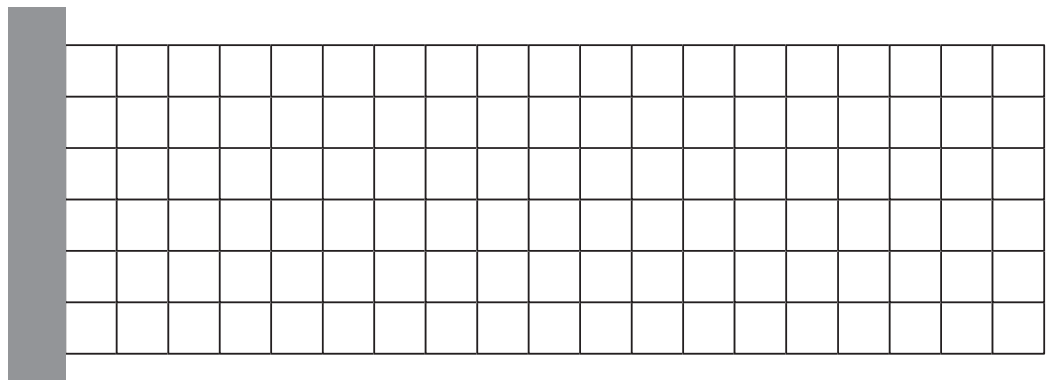

Fig. 6. Initial FE model for test plate (114 elements, 840 d.o.f.'s).

$1^{\text {st }}$ mode

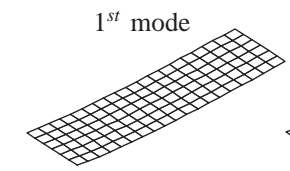

$2^{\text {nd }}$ mode

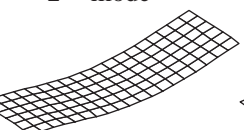

Fig. 7. Mode shapes of initial FE model. 
Table 1

Comparison of modal properties of cracked plate and initial FE model

\begin{tabular}{lllll}
\hline Mode & \multicolumn{2}{l}{ Natural frequency $(\mathrm{Hz})$} & \multirow{2}{*}{ MAC value } \\
\cline { 2 - 4 } & Test model & Initial FE model & Error $(\%)$ & \\
\hline 1 & 3.60 & 3.77 & 4.75 & 1.0000 \\
2 & 22.72 & 23.59 & 3.83 & $\mathbf{0 . 3 4 4 3}$ \\
3 & 23.71 & 24.55 & 3.53 & $\mathbf{0 . 5 1 0 0}$ \\
4 & 65.10 & 66.36 & 1.94 & 0.9501 \\
\hline
\end{tabular}

If a single-objective optimisation is used, then an objective function can be set up as

$$
\sum_{i=1}^{4} w_{f_{i}}\left|f_{x_{i}}-f_{a_{i}}\right|+\sum_{i=1}^{4} w_{m_{i}}\left(1-\operatorname{MAC}\left(\phi_{x_{i}}, \phi_{a_{i}}\right)\right)
$$

where $f_{x_{i}}, \phi_{x_{i}}$ are the $i$ th modal properties of the test plate, $f_{a_{i}}, \phi_{a_{i}}$ are the $i$ th modal properties of the FE model, and the coefficients $w_{f_{i}}, w_{m_{i}}$ are weighting factors. The selection of the weighting factors is very difficult since the relative importance among the objective terms is not obvious. The time-consuming optimisation process may be solved repeatedly until appropriate results are obtained. But if multiobjective optimisation is used, several objective terms can be minimised simultaneously and the solution in the non-convex surfaces can be generated. A multiobjective optimisation problem can be expressed as

$$
\operatorname{minimise}\left\{\sum_{i=1}^{4}\left|f_{x_{i}}-f_{a_{i}}\right|, 1-M A C_{11}, 1-M A C_{22}, 1-M A C_{33}, 1-M A C_{44}\right\}
$$

where $M A C_{i i}$ is $\operatorname{MAC}\left(\phi_{x_{i}}, \phi_{a_{i}}\right)$.

In the following two model updating cases, it is assumed that the mass matrix of the initial FE model is correct and only the stiffness matrix needs to be updated. The multiobjective optimisation problem defined in Eq. (7) is solved using the Pareto GA. Every updating parameter is arbitrarily bound between -0.5 and 0.5 .

\subsubsection{Case 1-three substructure parameters}

In this case, the FEs of the FE model are grouped into three substructures as in Fig. 8. The stiffness correction matrix is expressed as

$$
\Delta \boldsymbol{K}=\sum_{i=1}^{3} p_{k_{i}} \boldsymbol{K}_{i}
$$

where $\boldsymbol{K}_{i}$ is the stiffness matrix of the $i$ th substructure, and the coefficient $p_{k_{i}}$ is the updating parameter (substructure stiffness parameter). The multiobjective optimisation problem of Eq. (7) is solved and the statistics of the resulting Pareto-optimal solutions are listed in Table 2. Among the Pareto optimal, three solutions are selected which satisfy the following criteria and the modal 


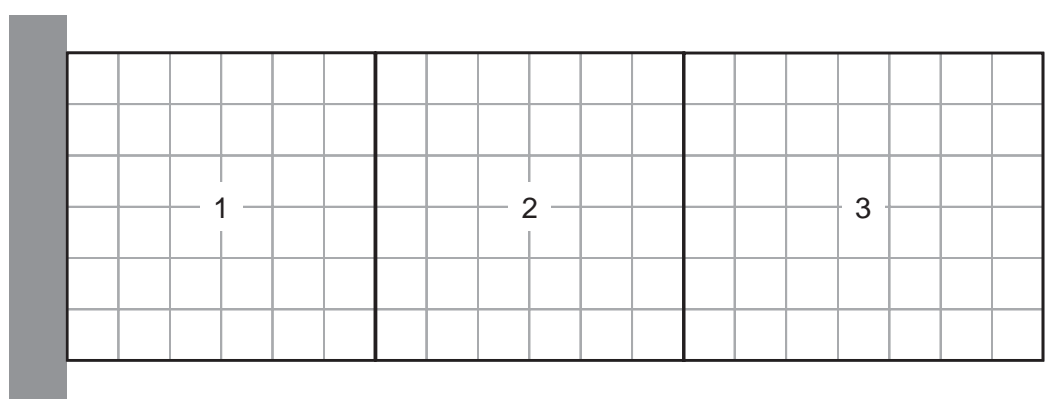

Fig. 8. Case 1-division of the plate model into three substructures.

Table 2

Case 1-statistics of Pareto-optimal solutions

\begin{tabular}{|c|c|c|c|c|c|c|}
\hline \multirow[t]{2}{*}{ Mode } & \multicolumn{3}{|c|}{ Natural frequency error $(\%)$} & \multicolumn{3}{|c|}{$M A C$ value } \\
\hline & Minimum & Maximum & Mean & Minimum & Maximum & Mean \\
\hline 1 & 0.0008 & 18.3963 & 4.0433 & 0.9993 & 1.0000 & 1.0000 \\
\hline 2 & 0.0129 & 21.3692 & 6.8310 & 0.3443 & 0.6547 & 0.3573 \\
\hline 3 & 0.0016 & 19.0698 & 4.9160 & 0.4849 & 0.5103 & 0.5094 \\
\hline 4 & 0.0028 & 19.3200 & 5.1409 & 0.9247 & 0.9508 & 0.9500 \\
\hline
\end{tabular}

Table 3

Case 1-comparison of modal properties of cracked plate and updated FE models

\begin{tabular}{|c|c|c|c|c|c|c|}
\hline \multirow[t]{2}{*}{ Mode } & \multicolumn{2}{|l|}{ Alternative $1^{\mathrm{a}}$} & \multicolumn{2}{|l|}{ Alternative $2^{\mathrm{b}}$} & \multicolumn{2}{|l|}{ Alternative $3^{\mathrm{c}}$} \\
\hline & $\begin{array}{l}\text { Natural freq. } \\
\text { error }(\%)\end{array}$ & $\begin{array}{l}M A C \\
\text { value }\end{array}$ & $\begin{array}{l}\text { Natural freq. } \\
\text { error }(\%)\end{array}$ & $\begin{array}{l}M A C \\
\text { value }\end{array}$ & $\begin{array}{l}\text { Natural freq. } \\
\text { error }(\%)\end{array}$ & $\begin{array}{l}M A C \\
\text { value }\end{array}$ \\
\hline 1 & 4.5000 & 0.9998 & 4.4902 & 0.9998 & 4.2901 & 0.9998 \\
\hline 2 & 4.5097 & 0.6547 & 4.5118 & 0.6547 & 4.5719 & 0.6547 \\
\hline 3 & 0.1616 & 0.4879 & 0.2363 & 0.4878 & 0.2054 & 0.4877 \\
\hline 4 & 0.7238 & 0.9479 & 1.4505 & 0.9469 & 1.7371 & 0.9468 \\
\hline
\end{tabular}

${ }^{\mathrm{a}} p_{k_{1}}=-0.2282, p_{k_{2}}=0.1602, p_{k_{3}}=0.1020$.

${ }^{\mathrm{b}} p_{k_{1}}=-0.2268, p_{k_{2}}=0.1462, p_{k_{3}}=0.1474$.

${ }^{\mathrm{c}} p_{k_{1}}=-0.2218, p_{k_{2}}=0.1354, p_{k_{3}}=0.1621$.

properties of the solutions are given in Table 3:

$$
\min _{i=1,2,3,4} M A C_{i i}>0.48 \text {. }
$$

Tables 2 and 3 show that the updated FE models do not give acceptable correlation with the test model. Also the updated parameters fail to have physically any meaning (Table 3 ). If the updated parameters are physically meaningful, $p_{k_{1}}$ should be some negative value since there is a crack in substructure 1 and the other two parameters, $p_{k_{2}}$ and $p_{k_{3}}$, should be close to zero. Thus, the model updating performed in case 1 is not successful. 


\subsubsection{Case 2-six substructure parameters}

In this case, the FEs of the FE model are grouped into six substructures as in Fig. 9. The stiffness correction matrix is expressed as

$$
\Delta \boldsymbol{K}=\sum_{i=1}^{6} p_{k_{i}} \boldsymbol{K}_{i} .
$$

Following the same procedure as in case 1, the multiobjective problem is solved and the statistics of the resulting Pareto-optimal solutions are listed in Table 4. Among the Pareto optimal, three solutions are selected which satisfy the following criteria and the modal properties of the solutions are given in Table 5:

$$
\min _{i=1,2,3,4} M A C_{i i}>0.98 \text {. }
$$

Unlike case 1, the updated FE models give quite acceptable correlation with the test model.

\subsubsection{Discussion}

Although both of the above two cases have an updating parameter in the region with the crack, $p_{k_{1}}$ for case 1 and $p_{k_{4}}$ for case 2, the initial FE model in case 1 are slightly improved in contrast with case 2. As noted, the improvement of a FE model depends heavily on the selection of parameters. This can be explained by examining the sensitivities of the objective functions with respect to each updating parameter. The sensitivities of $M A C_{22}$ and $M A C_{33}$ for both cases are

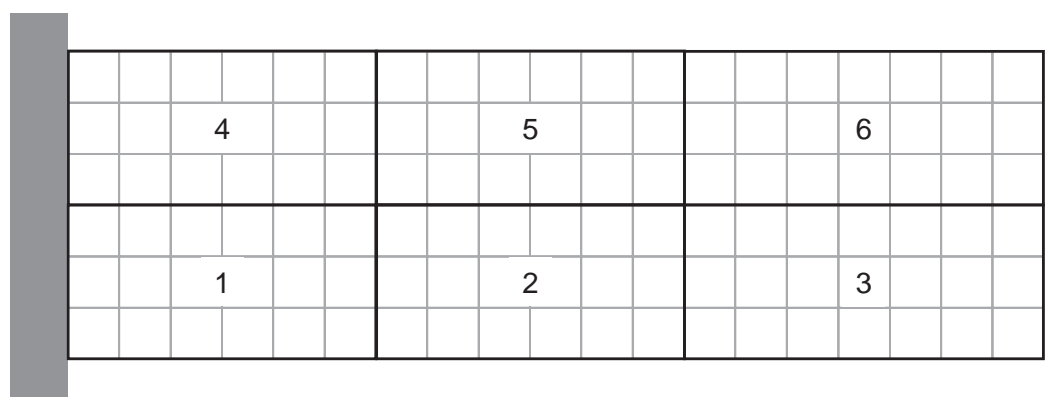

Fig. 9. Case 2-division of the plate model into six substructures.

\section{Table 4}

\begin{tabular}{|c|c|c|c|c|c|c|}
\hline \multirow[t]{2}{*}{ Mode } & \multicolumn{3}{|c|}{ Natural frequency error $(\%)$} & \multicolumn{3}{|c|}{$M A C$ value } \\
\hline & Minimum & Maximum & Mean & Minimum & Maximum & Mean \\
\hline 1 & 0.0018 & 14.4012 & 3.0504 & 0.9995 & 1.0000 & 0.9999 \\
\hline 2 & 0.0014 & 13.9952 & 3.2556 & 0.5499 & 0.9992 & 0.9543 \\
\hline 3 & 0.0042 & 16.1251 & 4.1042 & 0.6555 & 0.9991 & 0.9563 \\
\hline 4 & 0.0056 & 13.7168 & 2.6669 & 0.9487 & 0.9980 & 0.9920 \\
\hline
\end{tabular}

Case 2-statistics of Pareto-optimal solutions 
Table 5

Case 2 - comparison of modal properties of cracked plate and updated FE models

\begin{tabular}{|c|c|c|c|c|c|c|}
\hline \multirow[t]{2}{*}{ Mode } & \multicolumn{2}{|l|}{ Alternative $1^{\mathrm{a}}$} & \multicolumn{2}{|l|}{ Alternative $2^{\mathrm{b}}$} & \multicolumn{2}{|l|}{ Alternative $3^{\mathrm{c}}$} \\
\hline & $\begin{array}{l}\text { Natural freq. } \\
\text { error }(\%)\end{array}$ & $\begin{array}{l}M A C \\
\text { value }\end{array}$ & $\begin{array}{l}\text { Natural freq. } \\
\text { error }(\%)\end{array}$ & $\begin{array}{l}M A C \\
\text { value }\end{array}$ & $\begin{array}{l}\text { Natural freq. } \\
\text { error }(\%)\end{array}$ & $\begin{array}{l}M A C \\
\text { value }\end{array}$ \\
\hline 1 & 2.0380 & 0.9999 & 1.5040 & 0.9999 & 1.9387 & 0.9999 \\
\hline 2 & 1.2413 & 0.9817 & 1.2098 & 0.9843 & 2.5374 & 0.9882 \\
\hline 3 & 2.0865 & 0.9805 & 2.5083 & 0.9834 & 2.5510 & 0.9876 \\
\hline 4 & 0.9370 & 0.9922 & 0.4975 & 0.9927 & 1.0288 & 0.9910 \\
\hline
\end{tabular}

${ }^{\mathrm{a}} p_{k_{1}}=0.1681, p_{k_{2}}=-0.1968, p_{k_{3}}=0.4470, p_{k_{4}}=-0.4707, p_{k_{5}}=0.3474, p_{k_{6}}=-0.0478$.

${ }^{\mathrm{b}} p_{k_{1}}=0.2216, p_{k_{2}}=-0.2290, p_{k_{3}}=0.4105, p_{k_{4}}=-0.4969, p_{k_{5}}=0.4176, p_{k_{6}}=-0.0624$.

${ }^{\mathrm{c}} p_{k_{1}}=0.1383, p_{k_{2}}=-0.1558, p_{k_{3}}=-0.0111, p_{k_{4}}=-0.4562, p_{k_{5}}=0.4135, p_{k_{6}}=0.2802$.
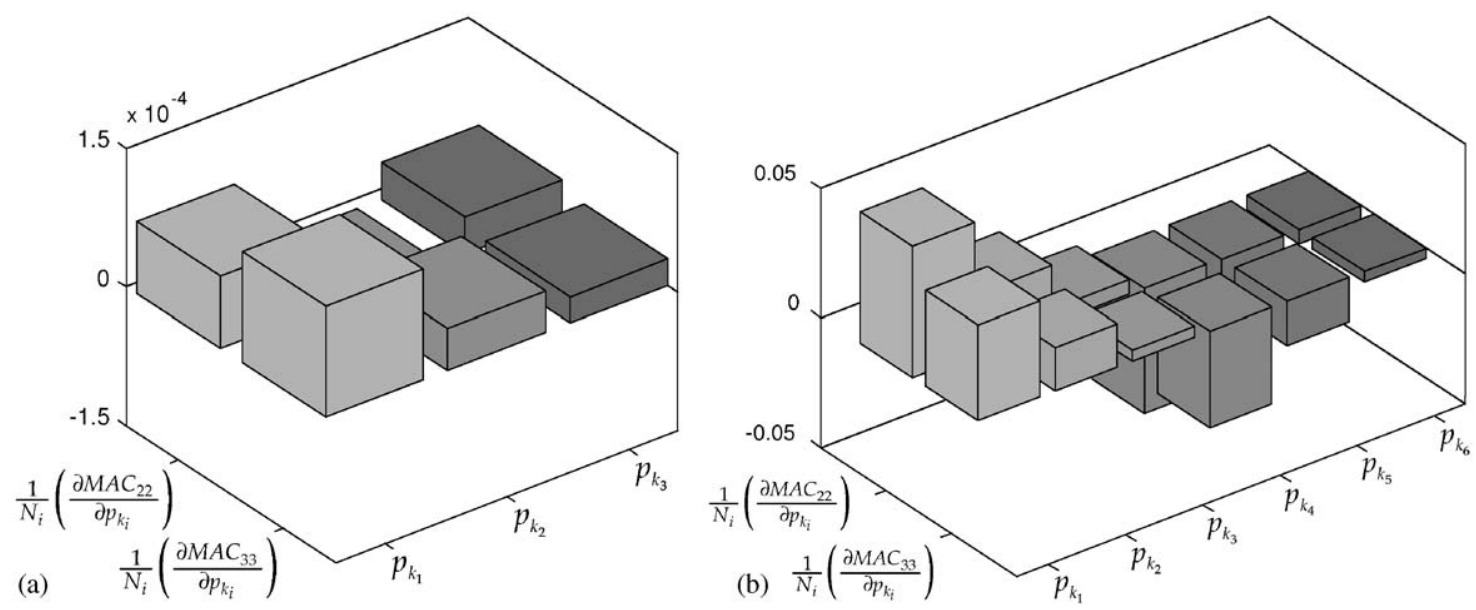

Fig. 10. Sensitivities of $M A C_{22}$ and $M A C_{33}$ with respect to the updating parameters $\left(N_{i}=\right.$ number of FEs in the $i$ th substructure): (a) case 1; (b) case 2.

plotted in Fig. 10. The sensitivities in case 2 (orders of $10^{-2}$ ) are much larger than those in case 1 (orders of $10^{-4}$ ). A successful FE model updating requires not only satisfactory correlation with experimental data but also minimum changes of an initial FE model. Thus, FEs of an FE model should be grouped into substructures such that the objective functions of primary interest are most sensitive to the updating parameters of the constructed substructures. But depending on the combinations of individual FEs, there exist numerous substructures or updating parameters. How can we select such an updating parameter set from many alternatives? A procedure is explained in the following to guide this selection.

\subsection{Updating parameter selection method}

Consider a substructure having $n$ individual FEs. Let $a$ be an updating parameter of the substructure and $a_{1}, a_{2}, \ldots, a_{n}$ be updating parameters of the individual FEs that constitute the 
substructure. Further assume that the updating parameters of both the substructure and the individual FEs have the same physical property: substructure parameter, Young's modulus, mass density, thickness, etc. An objective function value depends on the updating parameters and can be written as

$$
F(a) \text { or } F\left(a_{1}, a_{2}, \ldots, a_{n}\right) .
$$

Note $F(a)=F\left(a_{1}, a_{2}, \ldots, a_{n}\right)$ when $a_{i}=a$ for all $i=1,2, \ldots, n$. Using Taylor series expansion, the objective function can be written as

$$
F(a, a, \ldots, a)=F(0,0, \ldots, 0)+\left(\frac{\partial F}{\partial a_{1}}+\frac{\partial F}{\partial a_{2}}+\cdots+\frac{\partial F}{\partial a_{n}}\right) a+\cdots .
$$

Manipulating Eq. (13), we can obtain the sensitivity of the objective function with respect to the updating parameter $a$ as

$$
\frac{\partial F}{\partial a}=\lim _{a \rightarrow 0} \frac{F(a, a, \ldots, a)-F(0,0, \ldots, 0)}{a}=\frac{\partial F}{\partial a_{1}}+\frac{\partial F}{\partial a_{2}}+\cdots+\frac{\partial F}{\partial a_{n}}=\sum_{i=1}^{n} \frac{\partial F}{\partial a_{i}} .
$$

Eq. (14) tells that the sensitivity of an objective function with respect to an updating parameter of a substructure is equal to the sum of the sensitivities of the objective function with respect to the updating parameters of the individual FEs that constitute the substructure. Using this fact, we can construct substructures such that an objective function is sensitive to the updating parameters of the substructures as below:

(1) Calculate the sensitivity of the objective function about each individual FE.

(2) Group the individual FEs into substructures according to the calculated sensitivity signs considering the connectivity of FE mesh.

Fig. 11(a) schematically shows the updating parameter selection procedure for a singleobjective function. Using the suggested parameter selection method, the number of updating parameters can be kept small. And an objective function which quantifies the differences between analytical and experimental results can be reduced effectively with small changes of the selected updating parameters. Fig. 11(b) shows the parameter selection procedure when multiple objective functions are considered simultaneously. When multiple objective functions are considered simultaneously, trade-offs can be made in selecting parameters.

\section{Overall FE model updating procedure}

Using the Pareto GA and the parameter selection procedure, Pareto-optimal solutions with improved correlations with experimental data can be obtained. But for physically meaningful results, regions containing modelling errors should be identified and updating parameters should be selected around those regions. Examples of obvious candidates can be boundaries and joints. Also using error localisation methods, the parameters which presumably contain modelling errors can be located among a large candidate set of parameters. An FE model updating procedure 


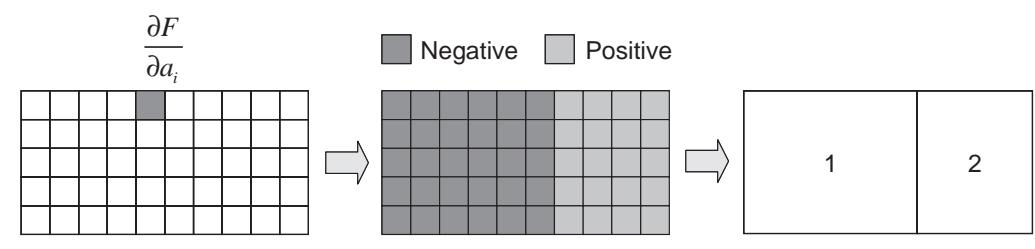

(a)
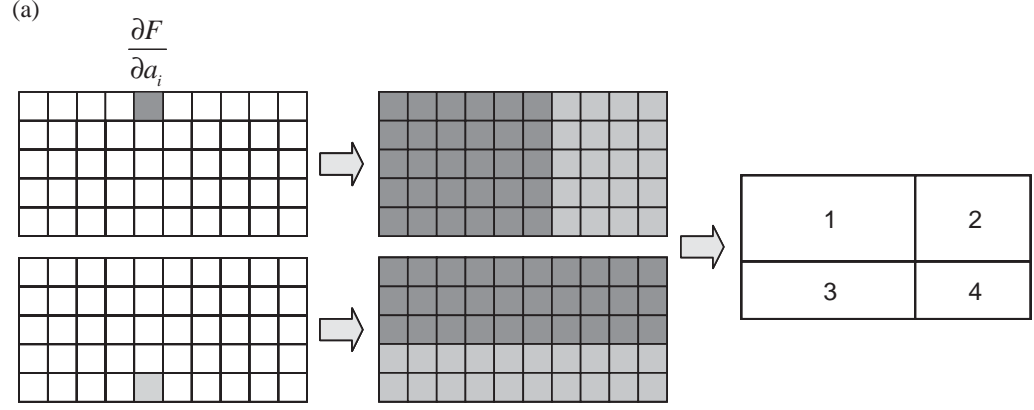

(b)

$$
\frac{\partial G}{\partial a_{i}}
$$

Fig. 11. Schematic of the updating parameter selection procedure: (a) for a single-objective function, (b) for multipleobjective functions.

incorporating error localisation is suggested as [10]:

(1) Set up a multiobjective function considering the correlations between analytical and experimental results.

(2) Locate modelling errors. This generally requires considerable physical insight. Systematic approaches such as error localisation methods can be used.

(3) The suggested parameter selection procedure is applied. First, select the most undesirable objective functions from the mulitobjective function. Then, calculate the sensitivities of the selected objective functions for each FE in the regions with modelling errors. And group the FEs into substructures such that the objective functions of primary interest are sensitive to the updating parameters of the substructures.

(4) Solve the multiobjective optimisation problem using the Pareto GA and select a best compromise solution from the Pareto-optimal solutions.

The suggested updating procedure is applied to the case study problem of Section 3.1. Using a force balance method [11], the regions with modelling errors are located as in Fig. 12. The plot shows dominant errors in the initial FE model around the crack position. For each FE in the dominant error regions, the sensitivities of $M A C_{22}$ and $M A C_{33}$ with respect to the chosen substructure stiffness parameter are calculated and the resulting signs are plotted in Fig. 13. In this special case, both sensitivities of each FE have the same sign. Using the sensitivity information, the elements are grouped into two substructures such that $M A C_{22}$ and $M A C_{33}$ are most sensitive to the updating parameters as in Fig 14. The stiffness correction matrix is written as

$$
\Delta \boldsymbol{K}=\sum_{i=1}^{2} p_{k_{i}} \boldsymbol{K}_{i}
$$




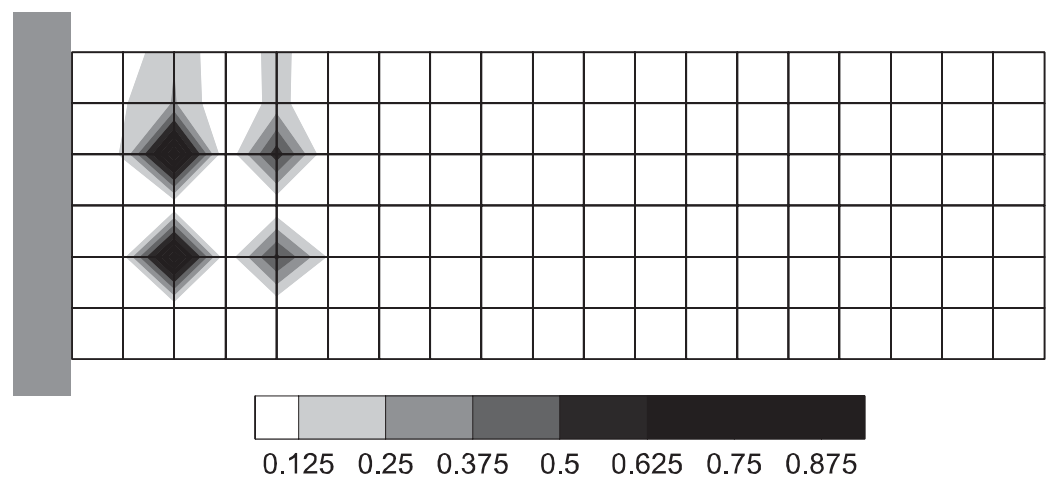

Fig. 12. Error localisation of the initial FE model using force balance method.

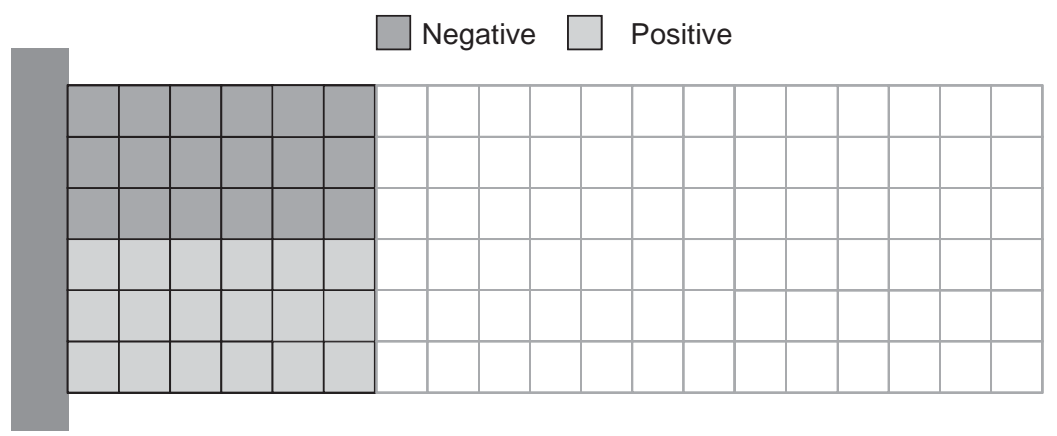

Fig. 13. Signs of $M A C_{22}$ and $M A C_{33}$ sensitivities.

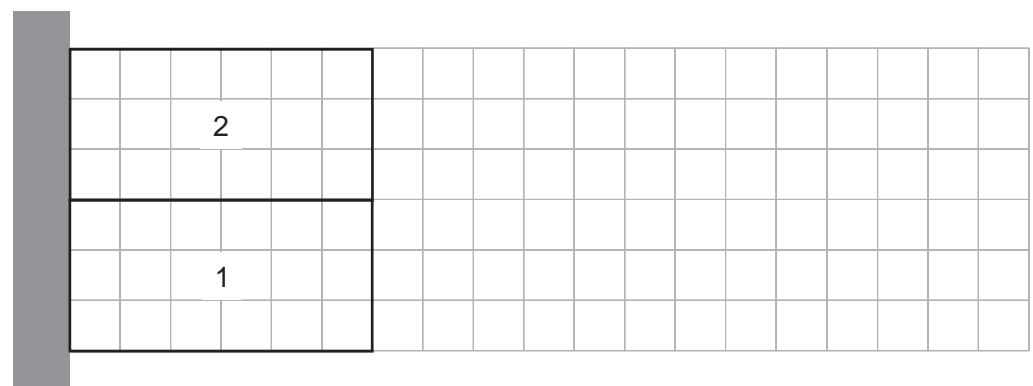

Fig. 14. Grouping of the regions with the dominant errors into two substructures.

The multiobjective optimisation problem of Eq. (7) is solved using the Pareto GA. The two updating parameters are arbitrarily bound between -0.7 and 0.7 . The statistics of the resulting Pareto-optimal solutions are listed in Table 6. And among the Pareto optimal, three solutions are selected which satisfy the following criteria and the modal properties of the selected solutions are given in Table 7:

$$
\min _{i=1,2,3,4} M A C_{i i}>0.97
$$


Table 6

Statistics of Pareto-optimal solutions

\begin{tabular}{|c|c|c|c|c|c|c|}
\hline \multirow[t]{2}{*}{ Mode } & \multicolumn{3}{|c|}{ Natural frequency error $(\%)$} & \multicolumn{3}{|c|}{$M A C$ value } \\
\hline & Minimum & Maximum & Mean & Minimum & Maximum & Mean \\
\hline 1 & 0.0000 & 14.3804 & 3.8505 & 0.9996 & 1.0000 & 0.9999 \\
\hline 2 & 0.0024 & 9.9437 & 2.2159 & 0.3040 & 0.9991 & 0.9373 \\
\hline 3 & 0.0000 & 6.2138 & 1.3152 & 0.4797 & 0.9987 & 0.9424 \\
\hline 4 & 0.0000 & 9.8404 & 3.0615 & 0.9469 & 0.9981 & 0.9944 \\
\hline
\end{tabular}

Table 7

Comparison of modal properties of cracked plate and updated FE models

\begin{tabular}{|c|c|c|c|c|c|c|}
\hline \multirow[t]{2}{*}{ Mode } & \multicolumn{2}{|l|}{ Alternative $1^{\mathrm{a}}$} & \multicolumn{2}{|l|}{ Alternative $2^{\mathrm{b}}$} & \multicolumn{2}{|l|}{ Alternative $3^{c}$} \\
\hline & $\begin{array}{l}\text { Natural freq. } \\
\text { error }(\%)\end{array}$ & $\begin{array}{l}M A C \\
\text { value }\end{array}$ & $\begin{array}{l}\text { Natural freq. } \\
\text { error }(\%)\end{array}$ & $\begin{array}{l}M A C \\
\text { value }\end{array}$ & $\begin{array}{l}\text { Natural freq. } \\
\text { error }(\%)\end{array}$ & $\begin{array}{l}M A C \\
\text { value }\end{array}$ \\
\hline 1 & 4.2457 & 0.9999 & 4.3283 & 0.9999 & 4.4387 & 0.9999 \\
\hline 2 & 3.2177 & 0.9700 & 3.2677 & 0.9706 & 3.3290 & 0.9713 \\
\hline 3 & 0.1638 & 0.9701 & 0.1230 & 0.9706 & 0.0659 & 0.9712 \\
\hline 4 & 4.1746 & 0.9911 & 4.2189 & 0.9910 & 4.2734 & 0.9909 \\
\hline
\end{tabular}

Tables 6 and 7 show that the updated FE models give quite acceptable correlations with the test model. Also, the updated parameters show consistent results with physical significance (Table 7). It is interesting that only two updating parameters can update the initial FE model successfully.

\section{Application}

The suggested model updating procedure is applied to FE model updating for a hard disk drive (HDD) cover structure as shown in Fig. 15 [12]. The initial FE model is developed on the basis of design geometries. Approximated thicknesses are used in the initial FE model because the actual HDD cover structure has tapered thickness and sometimes has abrupt changes in thickness. The thicknesses are measured only at several chosen points. The model has 1115 elements with 6732 d.o.f.'s. A modal test is conducted to obtain actual modal properties of the cover structure. The frequency range of interest is set from 0 to $3 \mathrm{kHZ}$ where high vibration and noise levels were observed during an operational test. The cover structure is excited with an impact hammer and vibrations are measured at 66 points using a laser doppler vibrometer. CADA-X is used to measure FRFs and extract mode shapes and natural frequencies. 


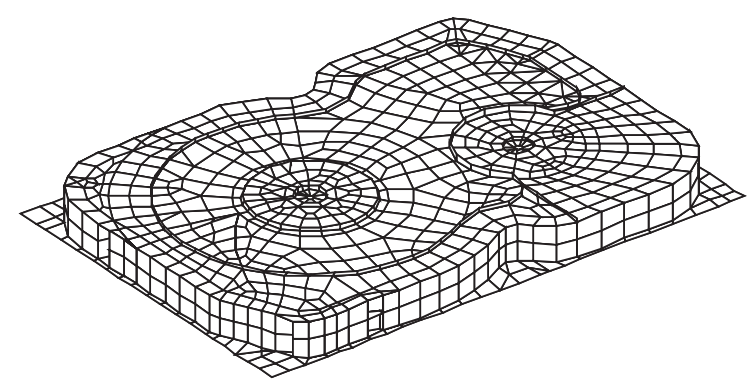

Fig. 15. Initial FE model of a HDD cover structure.

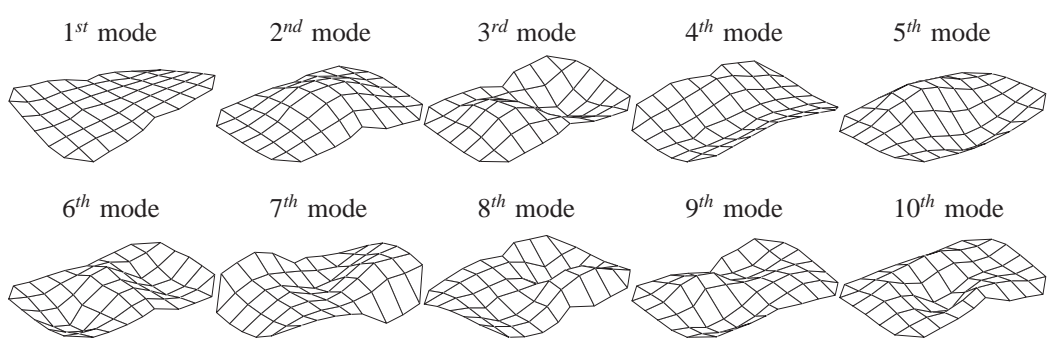

Fig. 16. Experimental mode shapes of HDD cover.

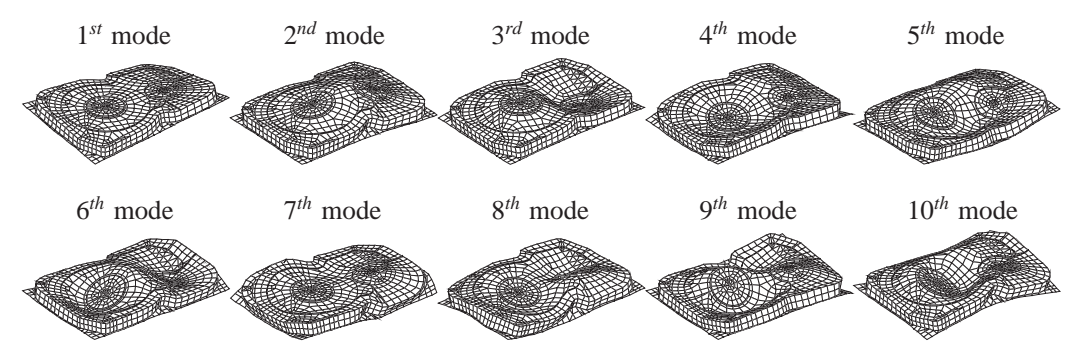

Fig. 17. Mode shapes of initial FE model.

The experimental mode shapes and their corresponding analytical mode shapes are shown in Figs. 16 and 17, respectively. And the modal properties are compared in Table 8. Table 8 shows that the natural frequency errors of the $6 \mathrm{th}, 7 \mathrm{th}, 8 \mathrm{th}$, and 9 th mode pairs are larger than $3 \%$. Also the $M A C$ values of $3 \mathrm{rd}$, 4th, 5th and 10 th mode pairs are below 0.9 . To improve these unsatisfactory correlations, the suggested model updating procedure is applied. A multiobjective function to be minimised is set as

$$
\left\{\left|\frac{f_{a_{6}}-f_{x_{6}}}{f_{x_{6}}}\right|,\left|\frac{f_{a_{7}}-f_{x_{7}}}{f_{x_{7}}}\right|,\left|\frac{f_{a_{8}}-f_{x_{8}}}{f_{x_{8}}}\right|,\left|\frac{f_{a_{9}}-f_{x_{9}}}{f_{x_{9}}}\right| 1-M A C_{33}, 1-M A C_{44}, 1-M A C_{55}, 1-M A C_{1010}\right\}
$$

where $f_{x_{i}}$ and $f_{a_{i}}$ denote the $i$ th experimental and analytical natural frequencies, respectively, and $M A C_{i i}$ is the $M A C$ value of the $i$ th mode pair. To prevent the other correlations from giving 
Table 8

Comparison of the experimental and analytical modal properties before updating

\begin{tabular}{lllll}
\hline Mode & \multicolumn{2}{l}{ Natural frequency $(\mathrm{Hz})$} & \multirow{2}{*}{ MAC value } \\
\cline { 2 - 4 } & Experiment & Initial FE model & Error $(\%)$ & 0.9847 \\
\hline 1 & 409.68 & 404.13 & -1.3507 & 0.9831 \\
2 & 908.15 & 931.94 & 2.6206 & $\mathbf{0 . 8 3 2 6}$ \\
3 & 1707.65 & 1669.00 & -2.2633 & $\mathbf{0 . 7 7 5 4}$ \\
4 & 1748.86 & 1709.13 & -2.2717 & $\mathbf{0 . 8 3 8 2}$ \\
5 & 1793.23 & 1757.94 & -1.9681 & 0.9496 \\
6 & 2474.99 & 2399.10 & $-\mathbf{3 . 0 6 6 3}$ & 0.9469 \\
7 & 2843.29 & 2723.27 & $-\mathbf{4 . 2 2 1 3}$ & 0.9360 \\
8 & 2976.06 & 2878.29 & $-\mathbf{3 . 2 8 5 3}$ & 0.9582 \\
9 & 3113.84 & 3016.39 & $-\mathbf{3 . 1 2 9 8}$ & $\mathbf{0 . 8 9 0 5}$ \\
10 & 3268.98 & 3182.76 & -2.6374 & \\
\hline
\end{tabular}

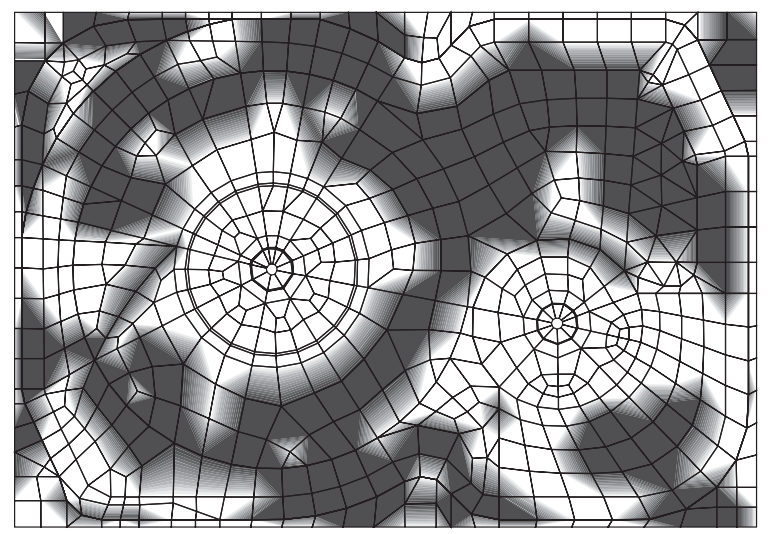

Fig. 18. Error localisation.

wrong optimisation results, several other constraints are applied as

$$
\left|\frac{f_{a_{i}}-f_{x_{i}}}{f_{x_{i}}}\right| \leqslant 3 \%, \quad i=1,2,3,4,5,10 \quad M A C_{i i} \geqslant 0.9, \quad i=1,2,6,7,8,9 .
$$

First, to find dominant error regions in the initial FE model, a force balance method is used [11] and the results are plotted in Fig. 18. By applying the suggested parameter selection method, 11 thickness parameters are selected around the suspicious regions as shown in Fig. 19. In selecting these parameters, the $2 \mathrm{nd}$, 5th, 6th, and 7 th objective functions, which show relatively large deviations, are considered altogether. And some trade-offs are made where there are competitions among them. Thus, the selected objective functions are very sensitive to the selected parameters. The multiobjective optimisation problem of Eq. (17) is solved using the Pareto GA. The allowed maximum change of the parameters is set to $5 \%$ (about $50 \mu \mathrm{m}$ ) considering only measurement error. The statistics of the solutions are summarised in Table 9. From the Pareto-optimal 


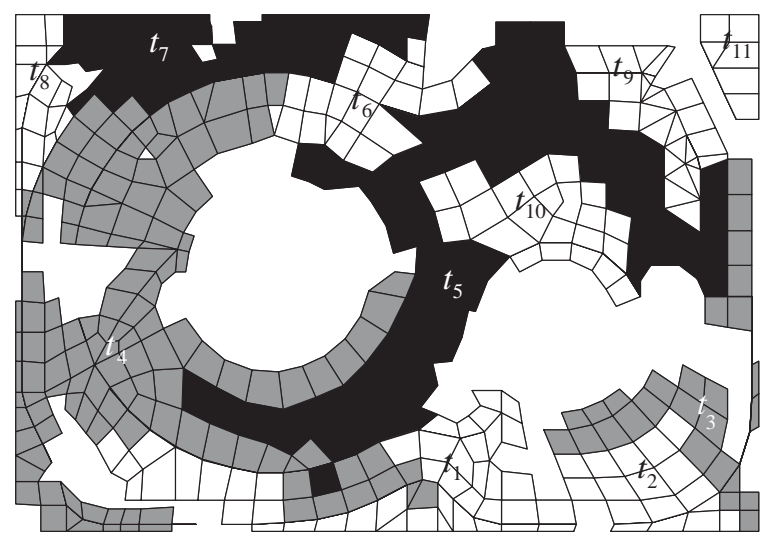

Fig. 19. Updating parameter selection: 11 thickness parameters.

Table 9

Statistics of Pareto-optimal solutions

\begin{tabular}{|c|c|c|c|c|c|c|}
\hline \multirow[t]{2}{*}{ Mode } & \multicolumn{3}{|c|}{ Natural frequency error $(\%)$} & \multicolumn{3}{|c|}{$M A C$ value } \\
\hline & Minimum & Maximum & Mean & Minimum & Maximum & Mean \\
\hline 1 & 0.0058 & 3.0095 & 1.2892 & 0.9816 & 0.9864 & 0.9846 \\
\hline 2 & 1.7950 & 3.2224 & 2.8667 & 0.9823 & 0.9852 & 0.9835 \\
\hline 3 & 1.1852 & 3.0775 & 1.8666 & 0.5800 & 0.9697 & 0.8950 \\
\hline 4 & 0.9975 & 3.0837 & 1.8269 & 0.3624 & 0.9835 & 0.8253 \\
\hline 5 & 0.9276 & 2.8706 & 1.9353 & 0.7706 & 0.9501 & 0.9112 \\
\hline 6 & 1.4276 & 3.9593 & 2.4784 & 0.9356 & 0.9626 & 0.9510 \\
\hline 7 & 2.5338 & 5.8786 & 3.7467 & 0.9005 & 0.9677 & 0.9499 \\
\hline 8 & 2.1765 & 4.0918 & 3.0111 & 0.9265 & 0.9613 & 0.9523 \\
\hline 9 & 1.5508 & 4.1371 & 2.5976 & 0.9038 & 0.9762 & 0.9666 \\
\hline 10 & 1.3372 & 3.0923 & 2.2688 & 0.8099 & 0.9414 & 0.9192 \\
\hline
\end{tabular}

solutions, three solutions are selected which satisfy the following criteria and their modal properties are listed in Table 10:

$$
\max _{i=1, \ldots, 10}\left|\frac{f_{a_{i}}-f_{x_{i}}}{f_{x_{i}}}\right| \leqslant 3 \%, \min _{i=1, \ldots, 10} M A C_{i i} \geqslant 0.9 .
$$

Table 10 shows the initial FE model considerably improved. For all the mode pairs, the natural frequency errors are less than $3 \%$, and the $M A C$ values are larger than 0.9. From these observations, it can be concluded that the FE model updating is successful.

\section{Conclusion}

Two important issues in model updating were considered in this paper: objective function and updating parameter selection. 
Table 10

Comparison of the experimental and analytical modal properties after updating

\begin{tabular}{|c|c|c|c|c|c|c|}
\hline \multirow[t]{2}{*}{ Mode } & \multicolumn{2}{|l|}{ Alternative 1} & \multicolumn{2}{|l|}{ Alternative 2} & \multicolumn{2}{|l|}{ Alternative 3} \\
\hline & $\begin{array}{l}\text { Natural freq. } \\
\text { error }(\%)\end{array}$ & $\begin{array}{l}M A C \\
\text { value }\end{array}$ & $\begin{array}{l}\text { Natural freq. } \\
\text { error }(\%)\end{array}$ & $\begin{array}{l}M A C \\
\text { value }\end{array}$ & $\begin{array}{l}\text { Natural freq. } \\
\text { error }(\%)\end{array}$ & $\begin{array}{l}M A C \\
\text { value }\end{array}$ \\
\hline 1 & 0.0119 & 0.9852 & 0.0125 & 0.9853 & 0.0121 & 0.9852 \\
\hline 2 & 0.0300 & 0.9834 & 0.0292 & 0.9835 & 0.0292 & 0.9834 \\
\hline 3 & 0.0148 & 0.9286 & 0.0150 & 0.9188 & 0.0153 & 0.9248 \\
\hline 4 & 0.0131 & 0.9090 & 0.0132 & 0.9027 & 0.0140 & 0.9046 \\
\hline 5 & 0.0204 & 0.9090 & 0.0216 & 0.9029 & 0.0214 & 0.9044 \\
\hline 6 & 0.0219 & 0.9510 & 0.0221 & 0.9506 & 0.0228 & 0.9514 \\
\hline 7 & 0.0286 & 0.9540 & 0.0289 & 0.9522 & 0.0292 & 0.9537 \\
\hline 8 & 0.0286 & 0.9545 & 0.0289 & 0.9535 & 0.0292 & 0.9545 \\
\hline 9 & 0.0248 & 0.9603 & 0.0260 & 0.9573 & 0.0247 & 0.9623 \\
\hline 10 & 0.0244 & 0.9090 & 0.0257 & 0.9025 & 0.0250 & 0.9122 \\
\hline
\end{tabular}

In conventional model updating using single-objective optimisation techniques, incompatible physical data are compared with each other using weighting factors. There are no general rules for selecting the weighting factors since the relative importance among the data is not obvious but specific for each problem. To avoid the difficulty, multiobjective optimisation was introduced in this paper. It was shown that multiobjective optimisation can optimise several objective functions simultaneously. A Pareto GA was especially used in this study. Note that the multiobjective GA is computationally expensive because it searches on the basis of a population of points, rather than a point-to-point search, and attempts to generate a large number of Pareto points. For a large problem where computational cost is important, other multiobjective techniques can be used.

Aware that the sensitivity of an objective function with respect to an updating parameter of a substructure is equal to the sum of the sensitivities of the objective function with respect to the updating parameters of the individual FEs, an improved updating parameter selection method was also suggested.

The two systematic approaches, multiobjective optimisation and parameter selection method, are seamlessly incorporated into a model updating procedure, which was successfully applied to a real engineering problem.

\section{Acknowledgements}

This work is supported by the project (NRL:M1-0001-00-0139) from the Ministry of Science and Technology, Korea.

\section{References}

[1] J.E. Mottershead, M.I. Friswell, Model updating in structural dynamics: a survey, Journal of Sound Vibration 162 (1993) 347-375. 
[2] M.I. Friswell, J.E. Mottershead, Finite Element Model Updating in Structural Dynamics, Kluwer Academic Publishers, Dordrecht, 1995.

[3] A. Berman, Validity of improved mathematical models, a commentary, Proceedings of the 16th International Modal Analysis Conference, Santa Babara, 1998, pp. 681-691.

[4] F.M. Hemez, Can model updating tell the truth? Proceedings of the 16th International Modal Analysis Conference, Santa Babara, 1998, pp. 1-17.

[5] R.I. Levin, N.A.J. Lieven, Dynamic finite element model updating using simulated annealing and genetic algorithms, Mechanical Systems and Signal Processing 9 (1995) 187-202.

[6] D.E. Goldberg, Genetic Algorithms in Search, Optimisation, and Machine Learning, Addison-Wesley, Reading, 1989.

[7] F.Y. Cheng, D. Li, Genetic algorithm development for multiobjective optimisation of structures, AIAA Journal 36 (1998) 1105-1112.

[8] M.J. Ratcliffe, N.A.J. Lieven, An improved method for parameter selection in finite element model updating, The Aeronautical Journal 102 (1995) 321-329.

[9] P. Avitabile, Model updating - endless possibilities, Proceedings of the 18th International Modal Analysis Conference, San Antonio, TX, 2000, pp. 562-570.

[10] G.H. Kim, Y.S. Park, Finite element model updating using multiobjective optimisation technique, Proceedings of the 19th International Modal Analysis Conference, Kimssimmee, FL, 2001, pp. 348-354.

[11] E. Fissette, C. Stavrinidis, S. Ibrahim, Error location and updating of analytical dynamic models using a force balance method, Proceedings of the 6th International Modal Analysis Conference, Kimssimmee, FL, 1988, pp. 1183-1190.

[12] G.H. Kim, Y.S. Park, Finite element model updating for a hard disk drive cover structure, Proceedings of the Asia-Pacific Vibration Conference, Hangzhou, China, 2001, pp. 255-258. 